\title{
Luminescence Depolarization Dynamics of Quantum Dots: Is It Hydrodynamic Rotation or Exciton Migration?
}

\author{
S. Shankara Narayanan, ${ }^{\dagger}$ Rupa Sarkar, ${ }^{\dagger}$ Sudarson Sekhar Sinha, ${ }^{\dagger}$ Fernando Dias, \\ Andy Monkman, ${ }^{\dagger}$ and Samir Kumar Pal*, ${ }^{*}$ \\ Unit for Nano Science \& Technology, Department of Chemical, Biological \& Macromolecular Sciences, \\ S. N. Bose National Centre for Basic Sciences, Block JD, Sector III, Salt Lake, Kolkata 700 098, India, and \\ Department of Physics, University of Durham, Durham, UK
}

Received: October 26, 2007; In Final Form: December 6, 2007

\begin{abstract}
In this paper, we report a first attempt to explore photoluminescence (PL) depolarization dynamics (anisotropy), which essentially depicts rotational motion of CdS quantum dots (QDs) of different sizes, by picosecondresolved time-correlated single-photon counting (TCSPC) and streak camera spectroscopic techniques. The possible interference of the internal exciton migration in the rotational motion of the QDs has been thoroughly investigated in the temperature range of 200-348 K. For the structural characterization of the QDs, optical absorption and emission spectroscopy, confocal microscopy, and dynamic light scattering (DLS) studies have been performed. The nature of the emission transition dipole of CdS QDs has also been examined by using polarization-gated steady-state emission spectroscopy. The present study demands an immense application in the field of bio-nanointerface to explore the hydrodynamic properties of a biological macromolecule.
\end{abstract}

\section{Introduction}

The dynamical properties of the local environment of a biomolecule are commonly evaluated by examining the orientations of transition dipoles (polarization/anisotropy) of an organic chromophore bound to a specific site of a protein/DNA. Timeresolved photoluminescence depolarization (anisotropy) is an excellent tool for monitoring the orientations of transition dipoles of an organic chromophore using the change in its emission anisotropy with time, and it has been used to provide information on the structural and dynamical rigidity in the vicinity of the chromophore. ${ }^{1}$ In addition to that, time-resolved anisotropy can also provide information on the hydrodynamic properties of a biomolecule. Molecular rotation of organic chromophores in liquids has been conventionally studied using a variety of techniques. ${ }^{2,3}$ Despite its widespread use, there are certain limitations of using organic chromophores for probing anisotropy and lifetime decay. For example, organic dye molecules contain linear dipoles that can provide information only about the in-plane orientation of the chromophores. Also, the shorter excited-state lifetime of an organic dye is a main obstacle for exploring the longer orientational relaxation processes of a biomolecule. Luminescent semiconductor quantum dots (QDs), a promising alternative to organic dyes for fluorescence-based applications such as immunoassays, in-situ hybridization, and long-term multicolor cell imaging, ${ }^{4,5}$ have also recently been utilized to unravel the dynamics of biomolecular interactions. ${ }^{6,7}$ The flexibility of these inorganic chromophores arises from the variability of the emission wavelength based on the size dependence of the quantum confinement effect. In contrast to common organic chromophores with a simple 1D transition dipole, QDs (such as CdSe) have been shown to possess a degenerate transition moment (2D transition moment) at cryo-

* Corresponding author. Fax: 91332335 3477. E-mail: skpal@bose.res.in.

$\dagger$ S. N. Bose National Centre for Basic Sciences.

$\div$ University of Durham. genic $^{8}$ as well as room temperature..$^{9-11}$ This $2 \mathrm{D}$ transition moment is inherently linked to the crystal structure ${ }^{10}$ and therefore allows the determination of the QD orientation via the analysis of the emission polarization anisotropy of single QDs. ${ }^{12}$ A 2D transition dipole, projected as an elliptical shape on the sample plane, intrinsically contains 3D orientation information ${ }^{9}$ that can provide information about the complex processes. Recently, Chung et al. ${ }^{9}$ employed a crossed polarizer setup to demonstrate the tracking of the 3D orientation of single CdSe QDs in a viscous environment. On the other hand, Schuster et al. ${ }^{13}$ used defocused wide-field fluorescence imaging to unravel the orientation and angular distribution of the emitted light of the 2D transition moment of CdSe/ZnS QDs. Although a few reports are in the literature that depict the measurement of a 2D emission transition dipole of QDs in solid films ${ }^{10,14}$ at room temperature, to the authors knowledge no reports are available regarding the measurement of a $2 \mathrm{D}$ transition dipole in the liquid phase. A few attempts ${ }^{11,15,16}$ to use QDs (with microsecond excited-state lifetime) as a probe for the detection of photoluminescence (PL) anisotropy have been made in this direction. However, due to the presence of various internal relaxation processes, it is difficult to recognize the dynamics of anisotropy of QDs merely in terms of its rotational depolarization. Exciton migration, ${ }^{17,18}$ which is well-known for semiconductor QDs, may coalesce with the rotational motion of the QDs and add complexity in data interpretation. In the present study, we demonstrate the presence of a $2 \mathrm{D}$ transition dipole for CdS QDs in the solution phase at room temperature, where biological applications can be pursued. ${ }^{6,19}$ We have also attempted to investigate the possible interference of the internal exciton migration process from the rotational motion of QDs. In the light of that fact, we have examined the role of temperature on the depolarization dynamics of the QDs. 


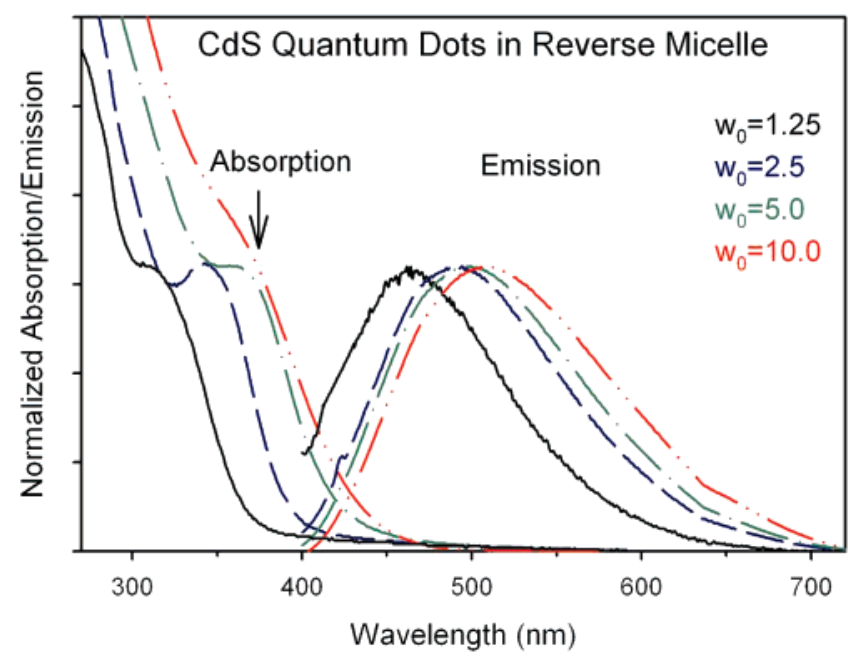

Figure 1. Normalized UV-vis absorption and photoluminescence (PL) spectra of CdS QDs in AOT/isooctane RMs with various values of $w_{0}$. The arrow in the figure indicates the excitation wavelength used for taking the emission spectra of CdS QDs.
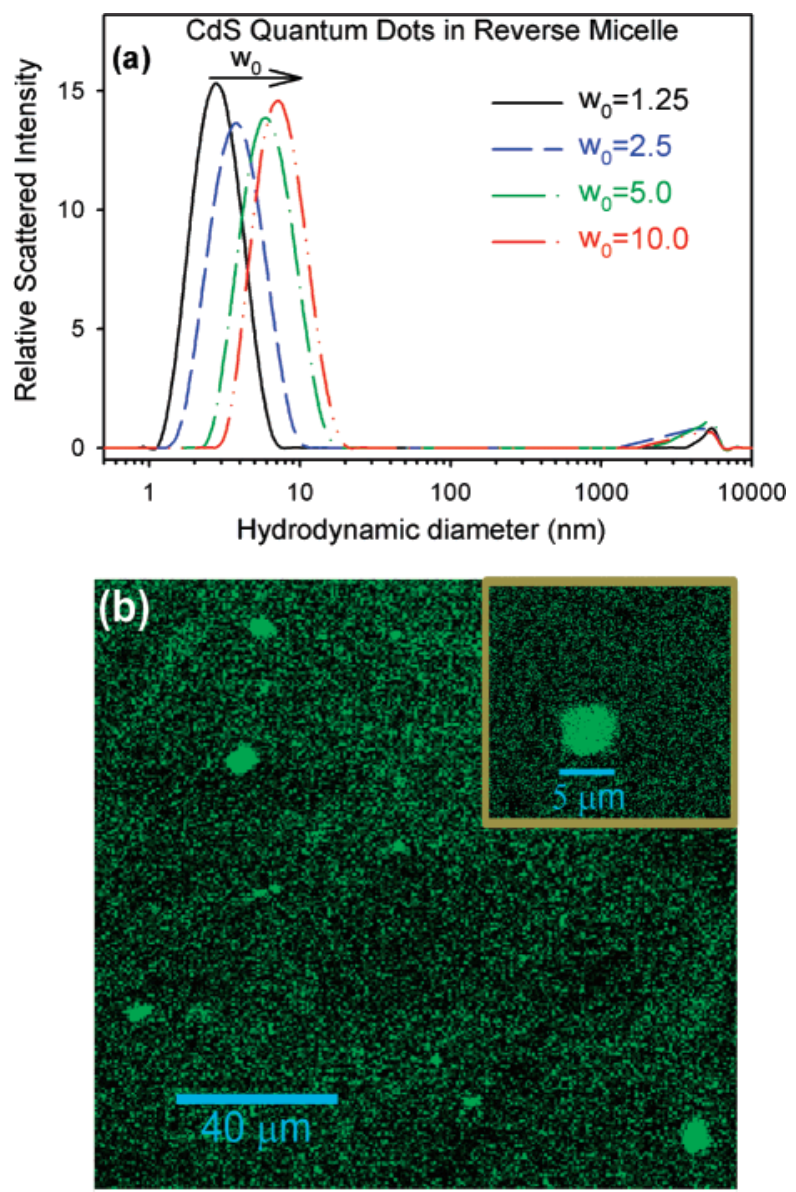

Figure 2. (a) Intensity-size distribution graph from dynamic light scattering (DLS) of CdS QDs in the RMs of $w_{0}=1.25,2.5,5$, and 10 at 295 K. (b) Confocal microscopy image of the QD film on a glass matrix. The inset shows larger QD aggregates at higher magnification.

\section{Materials and Methods}

Anhydrous $\mathrm{Na}_{2} \mathrm{~S} \cdot 9 \mathrm{H}_{2} \mathrm{O}, \mathrm{Cd}\left(\mathrm{NO}_{3}\right)_{2} \cdot 4 \mathrm{H}_{2} \mathrm{O}$, bis(2-ethylhexyl) sulfosuccinate sodium salt (AOT), 2,2,4-trimethylpentane (isooctane), and hexametaphosphate (HMP) were purchased from Sigma Aldrich. All the samples were used as received without further purifications. All aqueous solutions were prepared using
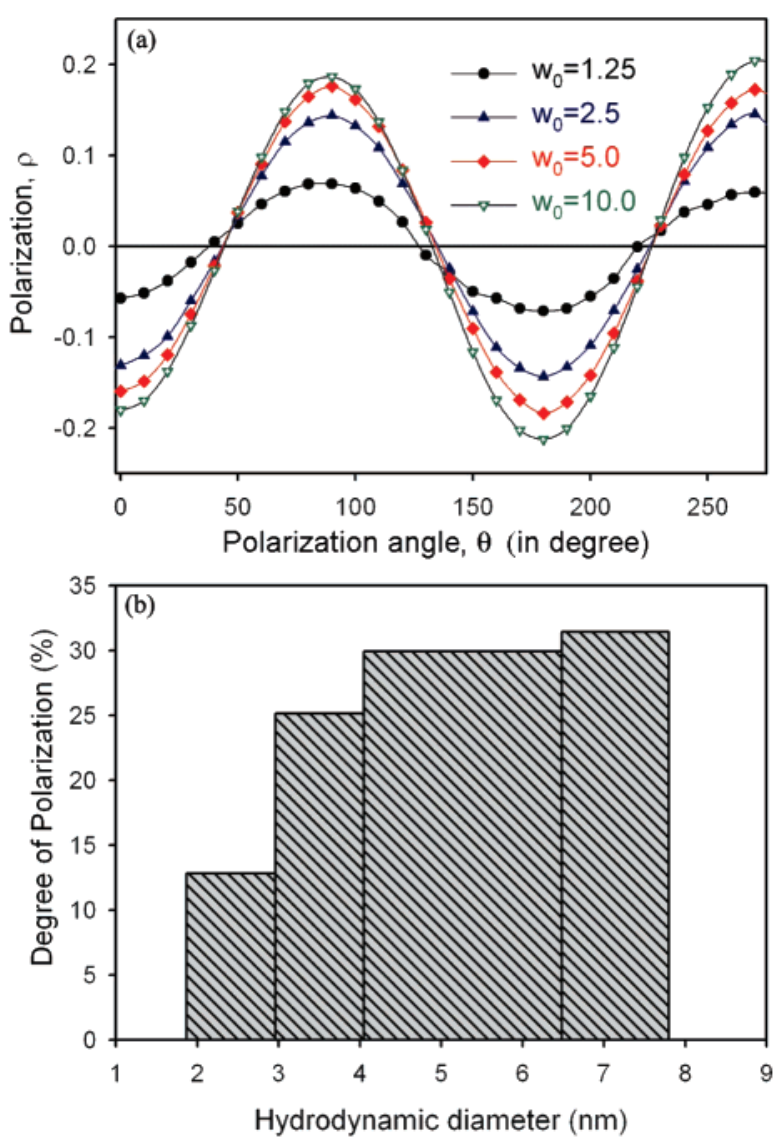

Figure 3. (a) Variation of the polarization value $(\rho)$ of CdS QDs of different sizes with the detected polarization angle. (b) The experimental distribution of the degree of polarization of CdS QDs having different hydrodynamic diameters.

double distilled water from a Millipore system. The synthesis of CdS QDs of various sizes was carried out ${ }^{20}$ by mixing two AOT reverse micellar $([\mathrm{AOT}]=166 \mathrm{mM})$ solutions having the same value of $w_{0}\left(w_{0}=\left[\mathrm{H}_{2} \mathrm{O}\right] /[\mathrm{AOT}]\right)$, one containing sulfide ions $\left(\mathrm{Na}_{2} \mathrm{~S}\right.$ in $100 \mathrm{mM}$ aqueous solution of $\left.\mathrm{HMP}\right)$ and the other in which cadmium ions $\left[\mathrm{Cd}\left(\mathrm{NO}_{3}\right)_{2}\right.$ in aqueous solution] were present. The mixing was produced by rapid injection of a variable volume of solutions of $\mathrm{Cd}^{+2}$ and $\mathrm{S}^{-2}$ of the same concentration. The final ratio of $\left[\mathrm{Cd}^{2+}\right] /\left[\mathrm{S}^{2-}\right]$ in the solution was maintained to be $2: 1$. For our experiments, we have synthesized CdS QDs of four different sizes $\left(w_{0}=1.25,2.5,5\right.$, and 10).

UV-vis absorption spectroscopy, steady-state photoluminescence (PL), and dynamic light scattering (DLS) were done using a Shimadzu UV-2450 spectrophotometer, a Jobin Yvon Fluoromax-3 fluorimeter, and a Malvern instruments Nano-S, respectively. A Leica TCS SPE spectral confocal system has been used for fluorescence microscopy. Steady-state polarization measurements have been carried out using the fluorimeter to identify the nature of the emission transition dipole for $\mathrm{CdS}$ QDs. The polarization, $\rho$, is defined as $\rho=\left(I_{\mathrm{vv}}-I_{\mathrm{va}}\right) /\left(I_{\mathrm{vv}}+\right.$ $I_{\mathrm{va}}$ ), where $a$ is defined by the polarization angle, $\theta$, in Figure 3a (i.e., $\theta=90^{\circ}$ implies $a=h$ ). DLS measurements are done employing a $4 \mathrm{~mW} \mathrm{He}-\mathrm{Ne}$ laser $(\lambda=632.8 \mathrm{~nm})$ equipped with a thermostatted sample chamber. All the scattered photons are collected at $173^{\circ}$ scattering angle. The scattering intensity data are processed using the instrumental software to obtain the hydrodynamic diameter $\left(d_{\mathrm{H}}\right)$ and the size distribution of the scatterer in each sample. The instrument measures the timedependent fluctuation in the intensity of light scattered from 
the particles in solution at a fixed scattering angle. The hydrodynamic diameter $\left(d_{\mathrm{H}}\right)$ of the reverse micelles is estimated from the intensity autocorrelation function of the time-dependent fluctuation in intensity, and $d_{\mathrm{H}}$ is defined as

$$
d_{\mathrm{H}}=\frac{k_{\mathrm{B}} T}{3 \pi \eta D}
$$

where $k_{\mathrm{B}}$ is the Boltzmann constant, $\eta$ is the viscosity, $T$ is the absolute temperature, and $D$ is the translational diffusion coefficient. In a typical size distribution graph from the DLS measurement, the $X$-axis shows a distribution of size classes in nanometers, while the $Y$-axis shows the relative intensity of the scattered light.

Time-Correlated Single Photon Counting Measurement (TCSPC). PL transients are measured and fitted by using a commercially available picosecond diode laser-pumped timeresolved fluorescence spectrophotometer (LifeSpec-ps) from Edinburgh Instruments [excitation wavelength $375 \mathrm{~nm}, 80 \mathrm{ps}$ instrument response function (IRF)] with a temperature controller attachment from Julabo (Model F32). All transients are taken by using a TCSPC technique. A micro-channel plate-photomultiplier tube (MCP-PMT, Hammamatsu) was used to detect the photoluminescence from the sample after dispersion through a grating monochromator. For all transients, the polarizer in the emission side is adjusted to be at $0^{\circ}\left(I_{\text {para }}\right)$ and $90^{\circ}\left(I_{\text {perp }}\right)$ with respect to the polarization axis of the excitation beam, and anisotropy, $r(t)$, is defined as

$$
r(t)=\frac{\left[I_{\text {para }}-G I_{\text {perp }}\right]}{\left[I_{\text {para }}+2 G I_{\text {perp }}\right]}
$$

The magnitude of $G$, the grating factor of the emission monochromator of the TCSPC system, is calculated by considering the nature of the emitting transition dipole of the QDs to be 2-fold degenerate (2D) (see below). The experimental value $^{9}$ of the degree-of-polarization of a $2 \mathrm{D}$ dipole, which is defined as $\left(I_{\max }-G I_{\min }\right) / I_{\max }$, is obtained to be 0.59 .

Streak Camera Measurements. In the streak camera measurements, samples are excited at $385 \mathrm{~nm}$ by a frequencydoubled Ti:Sapphire laser (Mira 900, Coherent) with an output pulse width of less than $2 \mathrm{ps}$ and a repetition rate of $76.3 \mathrm{MHz}$. Perpendicular to the excitation path, the sample emission is passed through a double subtractive monochromator (Acton Spectra Pro 2300i) and finally detected by the photocathode of a Hamamatsu streak camera (C5680). In these settings, the scattered excitation beam is observed with a half pulse width

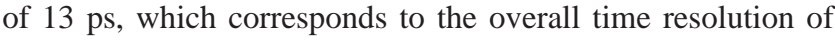
the setup. For the presented experiments, the sweep speed of the streak apparatus was adjusted to monitor sample emission as far as 700 ps. A spectral resolution of $4 \mathrm{~nm}$ has been determined using an argon calibration lamp. For the temperaturedependent studies, a cryostat (Janis) was attached to it.

Theory. The rotational correlation time $\left(\tau_{\mathrm{r}}\right)$ is calculated from the Stokes-Einstein-Debye (SED) theory ${ }^{11}$ and defined as

$$
\tau_{\mathrm{r}}=\frac{\eta V f C}{k_{\mathrm{B}} T}
$$

where $V$ is the volume of the solute molecule, $\eta$ is the shear viscosity of the solvent, $T$ is the absolute temperature, and $k_{\mathrm{B}}$ is the Boltzmann constant. The $f$ factor accounts for the shape of the solute molecule, approximated as a spheroid. $C$ is the solute-solvent coupling parameter that depends on the boundary conditions (either stick or slip) satisfied at the solute-solvent interface. For the stick limit, the first layer of the solvent sticks to the rotating solute, and the value of $C$ is equal to unity. For the slip limit, the solvent molecules on the rotating solute exert no tangential force and the $C$ value becomes nearly equal to zero.

The wobbling-in-cone model ${ }^{21,22}$ has been used to investigate the average angle of rotation due to inertial/ballistic motion and hydrodynamic rotation. The biexponential anisotropy decay can be analyzed using this two-step model. The faster rotational relaxation with shorter rotational time constant $\left(\tau_{\mathrm{f}}\right)$ is described as the motion of a restricted rotor (probe) having its transition dipole moment undergoing orientation diffusion within a semicone of angle $\theta_{\mathrm{w}}$ about an imaginary axis.

According to this model, the rotational anisotropy decay function is denoted as

$$
r(t)=r_{0}\left[\beta+(1-\beta) \exp \left(-\frac{\mathrm{t}}{\tau_{\mathrm{f}}}\right)\right] \exp \left(-\frac{\mathrm{t}}{\tau_{s}}\right)
$$

where $\tau_{\mathrm{s}}$ is the slow rotational relaxation with larger rotational time constant. Again, $\beta=S^{2}$, where $S^{2}$ is the generalized order parameter that describes the degree of restriction on the wobbling-in-cone orientational motion. $S^{2}$ satisfies the inequality in $0 \leq S^{2} \leq 1$, where $S^{2}=0$ describes unrestricted reorientation and $S^{2}=1$ means no wobbling-in-cone orientational motion. The semicone angle, $\theta_{\mathrm{w}}$, is obtained from the ordered parameter as follows:

$$
S^{2}=\left[\frac{1}{2} \cos \theta_{\mathrm{w}}\left(1+\cos \theta_{\mathrm{w}}\right)\right]^{2}
$$

\section{Results and Discussion}

The as-prepared CdS QDs in AOT/isooctane RMs with different $w_{0}$ values show characteristic optical absorption, photoluminescence (PL), and DLS signatures. Figure 1 shows $\mathrm{UV}-$ vis absorption spectra of the CdS QDs with various values of $w_{0}$. As is clear from the Figure 1, the excitonic peak as well as the absorption edge is shifted to shorter wavelength with the decrease in the value of $w_{0}$, indicating a decrease in the size of $\mathrm{QDs}^{20}$ due to quantum size effect. ${ }^{23}$ From the UV-vis absorption spectra, the mean diameter of the CdS QDs (in $\mathrm{nm}$ ) for each sample is either calculated from the first excitonic absorption peak ${ }^{24} \lambda_{\mathrm{m}}$ (in $\mathrm{nm}$ ) or the absorption edge $\lambda_{\mathrm{e}}{ }^{25}$ (in $\mathrm{nm})$ using the following equations:

$$
\begin{gathered}
2 R_{\mathrm{CdS}}\left(\lambda_{\mathrm{e}}\right)=0.1 /\left(0.1338-0.0002345 \lambda_{\mathrm{e}}\right) \\
2 R_{\mathrm{CdS}}\left(\lambda_{\mathrm{m}}\right)=\left(-6.6521 \times 10^{-8}\right) \lambda_{\mathrm{m}}{ }^{3}+ \\
\left(1.9557 \times 10^{-4}\right) \lambda_{\mathrm{m}}{ }^{2}-\left(9.2352 \times 10^{-2}\right) \lambda_{\mathrm{m}}+13.29
\end{gathered}
$$

The particle sizes estimated for $w_{0}=1.25,2.5,5$, and 10 are $1.1,1.9,3.8$, and $4.8 \mathrm{~nm}$, respectively, and the estimated band gaps using the effective mass model $^{26}$ are 4.4, 3.2, 2.7, and 2.6 $\mathrm{eV}$, respectively. The corresponding PL spectra of CdS QDs (right side of Figure 1) reveal emission maxima at 463, 491, 497, and $508 \mathrm{~nm}$ with increasing values of $w_{0}$, which are attributed to the recombination of charge carriers within surface states. However, the possibility of the emission from deep trap states cannot be completely ruled out.

The intensity distribution graph of the dynamic light scattering (DLS) of CdS QD with various values of $w_{0}$ at room temperature ( $295 \mathrm{~K}$ ) is shown in Figure 2a, revealing major scattering peaks at $2.96,4.05,6.48$, and $7.80 \mathrm{~nm}$. The signature of the $\sim 4 \mu \mathrm{m}$ cluster, i.e., larger aggregates, is also evident from DLS studies. With an increase in temperature from 273 to $348 \mathrm{~K}$, the diameter 
of RM containing CdS QDs with different $w_{0}$ values showed an insignificant change (data not shown). The PL from the individual QDs along with the signature of aggregated QDs is evident from the confocal microscopy image (Figure 2b). The inset of Figure $2 b$ shows one of those aggregates, which is also confirmed in the DLS experiment (Figure 2a). It should be noted that the diameter obtained from the absorption spectrum reveals the actual diameter of CdS QDs. However, DLS experiments give the hydrodynamic diameter, which is the overall diameter of CdS QDs along with the surfactant coating (AOT) used to stabilize it. According to the chemical structure of AOT, ${ }^{27}$ the linear length of the molecule is $12 \AA$ and the maximum crosssectional area is about $55 \AA .^{2}$ Considering the length of AOT molecules $(1.2 \mathrm{~nm})$ together with the size obtained from absorption spectra of CdS QDs in RMs, we obtain a good agreement with the hydrodynamic diameters obtained from DLS experiment for all values of $w_{0}$.

Evidence for the presence of 2D transition dipole moment in CdS QDs at $295 \mathrm{~K}$ is shown in Figure 3a. By sequentially rotating the emission polarizer from $0^{\circ}$ to $360^{\circ}$ and keeping the excitation polarizer at $0^{\circ}$, the PL intensity of the QDs is measured. The emission polarization values of the QDs of different sizes are plotted (Figure 3a) to show a sinusoidal pattern over rotated angles, which is similar to that obtained for 2D transition dipole of CdSe QDs. ${ }^{9}$ Figure 3a also depicts that, with the increase of the particle size, the steady-state emission polarization (equilibrium value) increases continuously. Figure $3 \mathrm{~b}$ demonstrates that the distribution of the degree of polarization values, defined as $\left[\left(I_{\max }-I_{\min }\right) / I_{\max }\right]$, at room temperature is very much consistent with a $2 \mathrm{D}$ transition dipole $^{8,9}$ of CdSe QDs. It is to be noted that no size-dependent polarization has been observed by Chung et al. ${ }^{9}$ in their farfield emission polarization microscopic experiments. However, the dependency of the emission polarization values on the size of the emitting CdS QDs is clearly evident (Figure 3) from our experiment. This apparent dissimilarity may be due to the difference in the state of the samples in these experiments. Chung et al. ${ }^{9}$ have measured a solid sample, where the possibility of rotational and translational motion of QDs are greatly reduced. Hence, the polarization values for different sized QDs show statistically the same distribution for the solid sample, whereas the polarization values in our experiment revealed different values due to the possible rotational and translational motions of the QDs in solution phase.

Figure $4 \mathrm{a}-\mathrm{c}$ reveals photoluminescence anisotropy decays of the QDs in $w_{0}=1.25 \mathrm{RM}$ at 273, 295, and $348 \mathrm{~K}$, respectively. The time-resolved anisotropy decays reveal the exponential nature with a decay time component of $\sim 2 \mathrm{~ns}$. With the change of temperature, the decay time constant alters. The DLS study reveals that the diameter of the RM $(2.96 \mathrm{~nm})$ containing the QDs remains unaltered in this temperature range. If isotropic diffusion and "stick" boundary conditions (where solvent molecules move with solute molecules) are considered, then the rotational correlation time is given by the SED equation. ${ }^{2,11}$ To obtain the theoretical estimate of the rotational correlation time $\left(\tau_{\mathrm{r}}\right)$, the temperature-dependent viscosity value of isooctane is taken from the literature ${ }^{28}$ and the calculated value of $\tau_{\mathrm{r}}$ is plotted against temperature (Figure $4 \mathrm{~d}$ ). The observed 2 ns component of the $r(t)$ decay fits well with the theoretical value (solid line of Figure $4 d$ ), revealing the origin of the time constant to be stick hydrodynamic rotation of the dots. The numerical fitting of the experimental data (Figure 4d) gives the average diameter of the QDs to be $3 \mathrm{~nm}$, which is in good agreement with DLS measurement (2.96 nm). Further, the
CdS Quantum dot in Reverse Micelle $\left(w_{0}=1.25\right)$
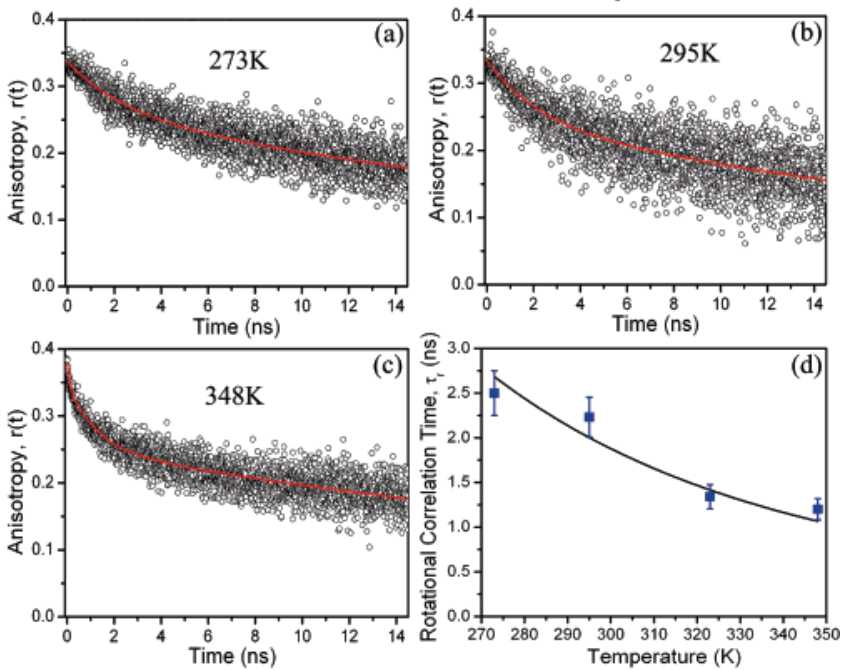

Figure 4. Time-resolved anisotropy decay of CdS QDs in RM of $w_{0}$ $=1.25$ obtained from TCSPC at (a) $273 \mathrm{~K}$ (b) $295 \mathrm{~K}$, and (c) $348 \mathrm{~K}$. (d) Plot of theoretical ( - ) and experimental ( $\mathbf{\square})$ rotational correlation time against temperature.
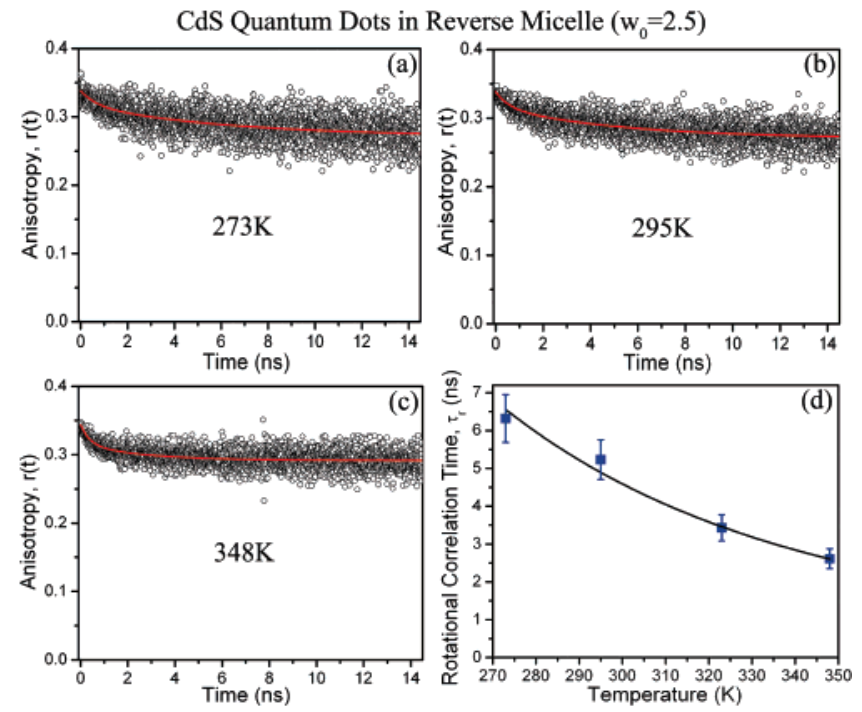

Figure 5. Time-resolved anisotropy decay of CdS QDs in RM of $w_{0}$ $=2.5$ obtained from TCSPC at (a) $273 \mathrm{~K}$ (b) $295 \mathrm{~K}$, and (c) $348 \mathrm{~K}$. (d) Plot of theoretical ( - ) and experimental (ם) rotational correlation time against temperature.

solute (RM with QD) is distinctly larger than the solvent isooctane, and the larger size $(3.00 \mathrm{~nm})$ of the solute guarantees that a greater amount of solvent displacement is needed for rotation of the RM revealing viscous environment. With the increase in temperature, the rotational correlation time becomes faster, due to the decrease in viscosity. At the highest experimental temperature $(348 \mathrm{~K})$, another faster decay time component ( $\sim 100 \mathrm{ps})$ arises that may be attributed to slip hydrodynamic rotation of the QD. The temperature-dependent anisotropy of larger-size QDs ( $w_{0}=2.5,5.0$, and 10.0) shows biexponential decay characteristics in the temperature range of $273-348 \mathrm{~K}$ (Figures 5and 6). Figure 5 depicts the temporal anisotropy decay of CdS QDs in RM of $w_{0}=2.5$, which reveal time constants of $\sim 0.4$ and $4 \mathrm{~ns}$ (Figure $5 \mathrm{a}-\mathrm{c}$ ). The longer time component fits well with the hydrodynamic stick boundary condition. On the other hand, the shorter time component of the anisotropy decay matches with the "slip" limit of the SED theory. For both these limits, the hydrodynamic diameter is calculated from the 

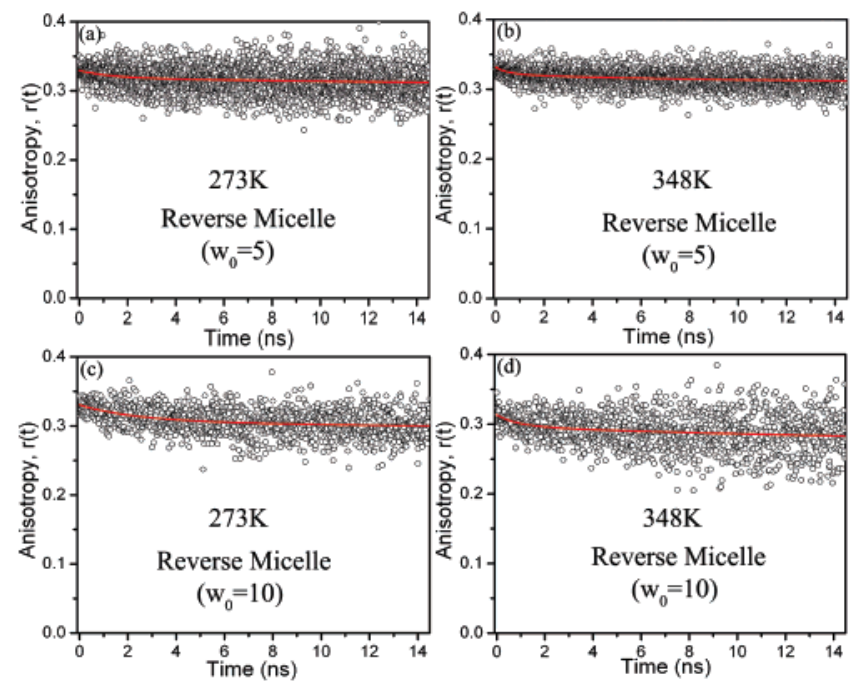

Figure 6. Time-resolved anisotropy decay of CdS QDs in (a and b) $w_{0}=5 \mathrm{RM}$ and (c and d) $w_{0}=10 \mathrm{RM}$ obtained from TCSPC at 273 and $348 \mathrm{~K}$.

numerical fitting of the experimental results (Figure 5d) and measured to be 4.04 and $4.09 \mathrm{~nm}$, which are in good agreement with the DLS measurements $(4.05 \mathrm{~nm})$. Figure 6, parts a and b, present time-resolved anisotropy decays of CdS QDs in $w_{0}=$ $5 \mathrm{RM}$ at 273 and $348 \mathrm{~K}$, respectively, which reveals similar time components. The similar biexponential anisotropy decay is also observed for CdS QDs in RM of $w_{0}=10$ (Figure 6c,d). It is to be noted that, with the increase in the particle size (from $w_{0}=1.25$ to 10 ), the rotational motion becomes slower and the offset value increases. The shorter and longer time components of QD of $w_{0}=5$ and 10 also follows the SED theory.

Experiments at higher time resolution have been performed using a streak camera with cryostat attachment to determine the presence of the missing component in the temperature range from 200 to $348 \mathrm{~K}$. Figure 7a shows a high-resolution streak camera anisotropy image of CdS QDs in $w_{0}=10 \mathrm{RM}$ at 295 $\mathrm{K}$. Wavelength-integrated time-resolved anisotropy depolarization (Figure 7b) illustrates the existence of a much shorter time component $(\sim 50 \mathrm{ps})$ in addition to the other two components obtained from TCSPC data. This orientational time may arise from the subslip hydrodynamic boundary condition, which is a clear indication of a weak solute-solvent interaction. The subslip behavior is plausible for nonpolar molecules in which no electronegative atoms are present. Hence, there is no scope for hydrogen bonding with solvent molecules that hinder the rotational motion. ${ }^{29}$ In the present study, RMs are suspended in isooctane, and there is no possibility of hydrogen bonding to take place. It should be noted that initial anisotropy $\left(r_{0}\right)$ decreases with decrease in temperature. We rationalize the observation in terms of the presence of increased scattered light from the sample at lower temperature even well away from the excitation wavelength, as the sample becomes solid with the lowering of temperature. However, the decay time constants are independent of the absolute value of $r_{0}$. The average rotational angle of $\mathrm{RM}^{30}$ with QD for slip hydrodynamic rotation changes from $\sim 20^{\circ}(273 \mathrm{~K})$ to $\sim 47^{\circ}(348 \mathrm{~K})$, whereas for stick hydrodynamic rotation, the rotational angle changes from $\sim 28^{\circ}$ $(273 \mathrm{~K})$ to $\sim 37^{\circ}(348 \mathrm{~K})$. The time-integrated anisotropy, which is identical to steady-state anisotropy, demonstrates that the anisotropy value remains the same at all the observation wavelengths, revealing monodispersity in size distribution (Figure 7c). At $200 \mathrm{~K}$, where all the rotational motions become

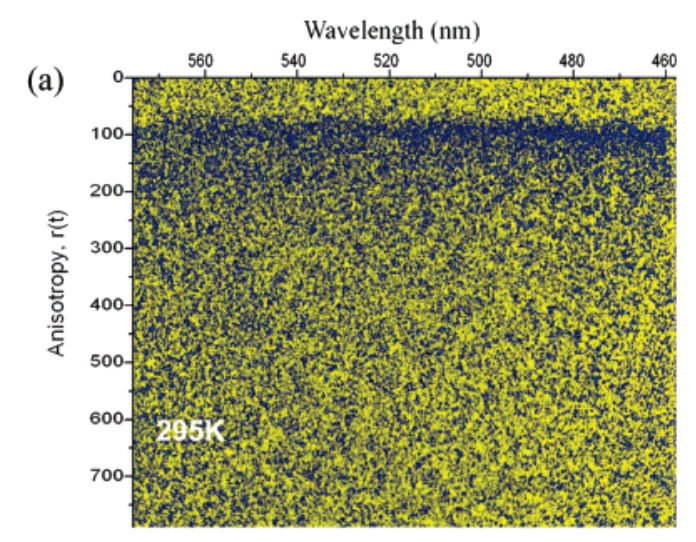

(b)
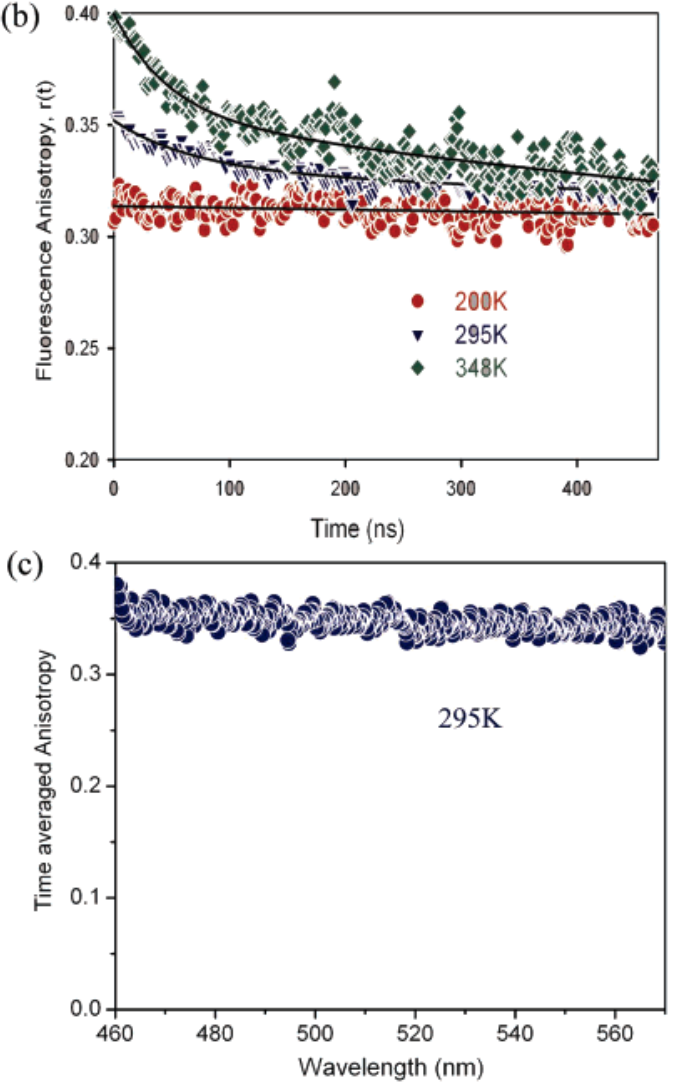

Figure 7. (a) High-resolution anisotropy image of CdS QDs in $w_{0}=$ 10 RM obtained from streak camera measurements at $295 \mathrm{~K}$. (b) Wavelength-dependent time-resolved anisotropy decay of CdS QDs in $w_{0}=10 \mathrm{RM}$ centered at $520 \mathrm{~nm}$ (time zero-corrected) at different temperatures. (c) Time integrated anisotropy of CdS QDs in $w_{0}=10$ $\mathrm{RM}$ at $295 \mathrm{~K}$.

frozen, no decay component in anisotropy is observed. It is true that the entire solution is frozen at $200 \mathrm{~K}\left(-73^{\circ} \mathrm{C}\right)$, although the freezing temperature of the bulk isooctane is $166 \mathrm{~K}(-107$ $\left.{ }^{\circ} \mathrm{C}\right)$. We anticipate that the elevation in the freezing point could be due to the presence of the AOT surfactant in the isooctane solution. Moreover, in the temperature the structural integrity of the RMs are also questionable. However, in our work we have considered the viscosity of the isooctane as the host solvent of the RM in the calculation, when the structural integrity has been maintained (from DLS data). For QDs, the electrons are known to migrate within different defect or trap states generated in it (i.e. intra-QD exciton migration). ${ }^{16,31} \mathrm{~A}$ small variation of site energy leads to hopping/migration of electron from one localized state to another. Upon trapping, the electron migrates to ever increasingly deep traps at the cost of thermal or kinetic 
energy, and electron migration from a shallower to deeper trap may be assisted by inhomogeneities of the bottom of the conduction band. The spatially anisotropic populations of excited QD formed by the absorption of polarized light may thus be diminished by the intra- and interdot energy transfer process, causing depolarization of PL. This exciton transfer is independent of the particle motion. A theoretical investigation ${ }^{17}$ of the luminescence polarization of CdSe microcrystal having hexagonal lattice structure provides information regarding the thermal/spin relaxation of electron/hole in the microcrystal and suggests that this process strongly requires the participation of different types of phonons. The study also depicts that, at low temperature, acoustic phonons give the main contribution to the depolarization process. Our low temperature $(200 \mathrm{~K})$ study rules out the possibility of the interference of collision-independent internal electronic properties of $\mathrm{QD}^{32}$ on the rotational motion of QDs.

\section{Conclusion}

In conclusion, CdS QDs synthesized in the AOT/isooctane RMs have been shown to possess a $2 \mathrm{D}$ dipole moment at room temperature using polarization-gated steady-state emission spectroscopy. Individual decay time components of the depolarization dynamics of CdS QDs in AOT/isooctane RMs have been classified as slip and stick hydrodynamic rotational motion. Due to slip motion, the QDs freely rotate through a significant angle between collisions that alter the magnitude or direction of its angular momentum. For both the stick and slip limits of rotational motion, the hydrodynamic diameter is calculated from the numerical fitting of the experimental results and found to be in good agreement with the size obtained from DLS measurements. Our study suggests that the depolarization pathway of QD is similar to that of an organic dye and QDs are able to report hydrodynamic rotation of a microenvironment. The internal dynamics or exciton migration does not interfere in the rotational anisotropy decay of QD.

Acknowledgment. S.S.N. and R.S. thank CSIR and UGC, respectively, for fellowships. We thank DST, India (SR/FTP/ PS-05/2004) and British Council (UKIERI) for financial grant.

\section{References and Notes}

(1) Pal, S. K.; Zewail, A. H. Chem. Rev. 2004, 104, 2099.

(2) Myers, A. B.; Pereira, M. A.; Holt, P. L.; Hochstrasser, R. M. J. Chem. Phys. 1987, 86, 5146.

(3) Zhang, Y.; Sluch, M. I.; Somoza, M. M.; Berg, M. A. J. Chem. Phys. 2001, 115, 4212.

(4) Jaiswal, J. K.; Mattoussi, H.; Mauro, J. M.; Simon, S. M. Nat. Biotechnol. 2003, 21, 47 .

(5) Zhao, M.; Beauregard, D. A.; Loizou, L.; Davletov, B.; Brindle, K. M. Nat. Med. 2001, 7, 1241.

(6) Larson, D. R.; Zipfel, W. R.; Williams, R. M.; Clark, S. W.; Bruchez, M. P.; Wise, F. W.; Webb, W. W. Science 2003, 300, 1434.

(7) Waybright, S. M.; Singleton, C. P.; Wachter, K.; Murphy, C. J.; Bunz, U. H. F. J. Am. Chem. Soc. 2001, 123, 1828.

(8) Sepiol, J.; Jasny, J.; Keller, J.; Wild, U. P. Chem. Phys. Lett. 1997, $273,444$.

(9) Chung, I.; Shimizu, K. T.; Bawendi, M. G. Proc. Natl. Acad. Sci. U.S.A. 2003, 100, 405 .

(10) Koberling, F.; Kolb, U.; Philipp, G.; Potapova, I.; Basche', T.; Mews, A. J. Phys. Chem. B 2003, 107, 7463.

(11) Petrov, E. P.; Cichos, F.; Zenkevich, E.; Starukhin, D.; Borczyskowski, C. V. Chem. Phys. Lett. 2005, 402, 233.

(12) Efros, A. L.; Rodina, A. V. Phys. Rev. B 1993, 47, 10005.

(13) Schuster, R.; Barth, M.; Gruber, A.; Cichos, F. Chem. Phys. Lett. 2005, 413, 280.

(14) Empedocles, S. A.; Neuhauser, R.; Bawendi, M. G. Nature 1999, 399, 126.

(15) Empedocles, S. A.; Bawendi, M. G. Science 1997, 278, 2114.

(16) Sarkar, R.; Shaw, A. K.; Narayanan, S. S.; Rothe, C.; Hintschich, S.; Monkman, A.; Pal, S. K. Opt. Mat. 2007, 29, 1310.

(17) Efros, A. L. Phys. Rev. B 1992, 46, 7448

(18) Rodt, S.; Türck, V.; Heitz, R.; Guffarth, F.; Engelhardt, R.; Pohl, U. W.; Stra $\beta$ burg, M.; Dworzak, M.; Hoffmann, A.; Bimberg, D. Phys. Rev. B 2003, 67, 235327.

(19) Dahan, M.; Laurence, T.; Pinaud, F.; Chemla, D. S.; Alivisatos, A. P.; Sauer, M.; Weiss, S. Opt. Lett. 2001, 26, 825.

(20) Petit, C.; Lixon, P.; Pileni, M. P. J. Phys. Chem. 1990, 94, 1598.

(21) Lipari, G.; Szabo, A. Biophys. J. 1980, 30, 489.

(22) Wang, C. C.; Pecora, R. J. Chem. Phys. 1980, 72, 5333.

(23) Brus, L. E. J. Chem. Phys. 1983, 79, 5566.

(24) Yu, W. W.; Qu, L.; Guo, W.; Peng, X. Chem. Mater. 2003, 15, 2854

(25) Moffitt, M.; Vali, H.; Eisenberg, A. Chem. Mater. 1998, 10, 1021.

(26) Brus, L. E. J. Chem. Phys. 1984, 80, 4403.

(27) Eicke, H. F.; Arnold, V. J. Colloid Interface Sci. 1974, 46, 101.

(28) Padua, A. A. H.; Fareleira, J. M. N. A.; Calado, J. C. G. J. Chem Eng. Data 1996, 41, 1488.

(29) Inamdar, S. R.; Mannekutla, J. R.; Mulimani, B. G.; Savadatti, M. I. Chem. Phys. Lett. 2006, 429, 141

(30) Shaw, A. K.; Pal, S. K. J. Phys. Chem. B 2007, 111, 4189.

(31) Burda, C.; Chen, X.; Narayanan, R.; El-Sayed, M. A. Chem. Rev. 2005, 105, 1025 .

(32) Rice, S. A. J. Phys. Chem. 1980, 84, 1280. 